\title{
Better Best Systems - Too Good To Be True ${ }^{1}$
}

\author{
Marius BACKMANN ${ }^{2}$ and Alexander REUTLINGER ${ }^{3}$
}

FORTHCOMING IN DIALECTICA

\begin{abstract}
Craig Callender, Jonathan Cohen and Markus Schrenk have recently argued for an amended version of the best system account of laws - the better best system account (BBSA). This account of lawhood is supposed to account for laws in the special sciences, among other desiderata. Unlike David Lewis's original best system account of laws, the BBSA does not rely on a privileged class of natural predicates, in terms of which the best system is formulated. According to the BBSA, a contingently true generalization is a law of a special science $\mathrm{S}$ iff the generalization is an axiom (or a theorem) of the best system relative to the set of predicates used by special science S. We argue that the BBSA is, at best, an incomplete theory of special science laws, as it does not account for typical features of special science laws, such as attached ceteris paribus conditions and the idealized character of law statements in these disciplines.
\end{abstract}

\footnotetext{
${ }^{1}$ This paper is fully co-authored.

${ }^{2}$ University of Konstanz, Email: marius.backmann@uni-konstanz.de

${ }^{3}$ Munich Center for Mathematical Philosophy, LMU Munich, Email: Alexander.Reutlinger@lmu.de
} 


\section{Introduction: Best Systems for the Special Sciences}

Craig Callender, Jonathan Cohen, and Markus Schrenk have recently presented an attempt to extend and, thereby, improve the best system account (henceforth, BSA) of laws (Lewis, 1973, 1994). ${ }^{4}$ They propose a "better best systems account" (henceforth, BBSA) of laws in physics as well as in the special sciences (Schrenk, 2007, 2008; Cohen and Callender, 2009, 2010). The BBSA is advocated in order to avoid several of the problems that the original BSA faces. We will be primarily concerned with evaluating whether BBSA is indeed successful in providing an account of special sciences laws. We suggest a negative evaluation.

We will proceed as follows: in section 2 we give a brief overview of the original best system account and its shortcomings motivating the BBSA. In section 3, we present the BBSA itself. In the central section 4, we discuss two challenges to the BBSA. These challenges demonstrate that BBSA does not provide what it is intended to do in the first place: an adequate theory of special science laws. Our first challenge consists in the claim that the BBSA by itself does not satisfy a criterion of adequacy for an account of special science laws (i.e. avoiding Lange's dilemma), and it is questionable whether the BBSA can easily be strengthened by standard procedures (that is, accounts of ceteris paribus conditions) to avoid this dilemma. The second challenge is that the BBSA has difficulties to accommodate idealized laws, which the majority of special science laws are. A theory of laws of nature that is particularly designed to account for special science laws and which is not applicable to the majority of these laws fails to serve its purpose.

\footnotetext{
4 Another recent amendment of the classical best system account - the statistical mechanical approach (Albert, 2000; Loewer, 2009) - will not be discussed in this paper, because the BBSA and the statistical mechanical approach disagree on too many substantial points.
} 


\section{The Original Best System Account}

The common core of different versions of the best system account is the claim that laws are a specific subset of the contingently true generalizations about regularities in nature. According to the original BSA advocated by David Lewis (1973, 73; 1994, 231-232), lawful generalizations can be distinguished from accidentally true generalizations because only the former are axioms or theorems of the best deductive system. A system is the best system if it optimizes the balance between simplicity and strength. ${ }^{5}$

Callender, Cohen and Schrenk praise the original BSA for its ability to distinguish accidental regularities from laws, to explain how laws support counterfactuals, to make sense of how laws are explanatory, to render nomic facts epistemically accessible, for its compatibility with objective probabilities etc. (Cohen and Callender 2009, 3). However as Cohen, Callender, and Schrenk observe - the original BSA has its own share of problems. We are aware of the fact that a number of objections have been raised against the BSA. For instance, it is controversial whether balance, strength, and simplicity can be defined precisely and according to which algorithm they trade off against one another (cf. van Fraassen 1989, 55-64 for a classic critique). We leave these objections aside because they pose a general problem for the BBSA and BSA alike. We focus on desiderata genuine to the BBSA.

To get a proper grasp on the BBSA, we focus on the two problems of the original BSA that Cohen and Callender present because they use these problems in order to motivate the BBSA, and because the second problem matters for our own objections to BBSA. Callender and Cohen identify the following two main problems for the original

${ }^{5}$ Lewis (1994: 233-236) applies the BSA to probabilistic laws and chances, which we will ignore for brevity's sake. 
BSA.

First: the problem of immanent comparisons. Cohen and Callender (2009, 5-8) diagnose a challenge to the BSA, which they call the problem of immanent comparisons - a problem already noticed by Lewis (1983), van Fraassen (1989), and Loewer (1996, 2007). The problem of immanent comparisons arises because simplicity, strength, and balance can only be determined relative to the language in which a system is formulated. This problem is most obvious for the case of simplicity: how simple a system is taken to be depends on the predicates used to formulate the sentences of which the system consists. Barry Loewer illustrates the language-dependent simplicity of a best system by using Nelson Goodman's famous predicate 'grue':

Simplicity, being partly syntactical, is sensitive to the language in which a theory is formulated, and so different choices of simple predicates can lead to different verdicts concerning simplicity. A language that contains 'grue' and 'bleen' as simple predicates but not 'green' will count 'All emeralds are green' as more complex than will a language that contains 'green' as a simple predicate. (Loewer 1996, 109)

Following Loewer and adopting Quinean terminology, Cohen and Callender call simplicity an immanent criterion, since simplicity can only be defined in terms of a specific language. There are no language-independent, transcendent criteria, according to which one might judge the simplicity of a system. Hence, two systems formulated in terms of different set of predicates cannot be compared regarding simplicity. Likewise, 
Callender and Cohen argue, strength is also an immanent criterion.

If there are no language-independent criteria, with respect to which one can compare rival systems, then it is not possible to compare one of them with another. This problem can be avoided if it is possible to prescribe a common language, in which to formulate all best systems. Lewis $(1983,42)$ argues for adopting a language containing only perfectly natural predicates. Then, inter-system comparison becomes possible because - due to a shared language - all rival systems share the same notions of simplicity, strength and balance. For Lewis, positing natural properties solves the problem of immanent comparison.

Second: the problem of supervenient properties. Cohen and Callender discuss the problem of supervenient properties, which consists in the charge that the original BSA fails to account for the special science laws (Cohen and Callender 2009, 14-15). Cohen and Callender argue that special sciences use predicates that are not perfectly natural and fundamental. They discuss two examples, one from statistical mechanics and one from the life sciences. First, entropy is a macroscopic property of physical systems (such as gases and fluids) as entropy supervenes on fundamental properties, and entropy figures in the second law of thermodynamics. Second, laws of the life sciences (for instance, the Volterra rule and the Bergmann rule) refer to not perfectly natural properties such as being a warm-blooded vertebrate, being prey, and so forth.

Why are properties such as entropy and being a warm-blooded a problem for the BSA? If the original BSA is coupled with a theory of natural predicates and properties, then the concept of, for instance, entropy cannot figure in a theorem or axiom of the best deductive system. Cohen and Callender argue that non-natural predicates cannot figure in 
statements of the best system for two reasons: either statements formulated in term of non-natural predicates are redundant (Cohen and Callender 2009, 10); or, if statements formulated in terms of non-natural predicates are translated into statements formulated in terms of natural predicates, the translation would be long, complicated, and disjunctive for instance, a list of all possible micro-realizers of an entropic macro-state of a gas (ibid.). Because of the long, complicated, and disjunctive translation macro-predicates would significantly compromise the simplicity of the deductive system in question. Hence, due to redundancy or lack of simplicity, Cohen and Callender argue, special science generalizations cannot be accepted as axioms of a best system according to the original BSA. ${ }^{6}$

\section{From Best Systems to Better Best Systems}

According to Callender, Cohen and Schrenk, Lewis simply made a mistake in requiring that the sentences of all best systems have to be formulated in terms of a once and for all fixed set of perfectly natural predicates. The guiding idea of the BBSA is that one is not committed to a singular choice of predicates. Instead there are many sets of predicates and a deductive system can be formulated relative to each of these sets. For instance, if one is interested in a theory of the laws of biology and economics, then one simply chooses different sets of predicates - a set $\mathbf{B}$ of biological predicates and a set $\mathbf{E}$ of

\footnotetext{
${ }^{6}$ Following Lewis (1983, 42), Schrenk (manuscript) discusses the possibility of lessthan-perfectly natural properties (such as 'being a tiger'). However, even if there were degrees of naturalness this would not solve the problem of supervenient properties. Although 'being a tiger' might have some degree of naturalness, 'being a tiger' could not figure in the best system formulated in terms of perfectly natural predicates for the reasons that Cohen and Callender outline: this predicate is either redundant or it is disqualified because it immensely complicates the system in question.
} 
economic predicates. A law of biology is a contingently true generalization that is a sentence in the best system stated in terms of the biological predicates. A law of economics is a contingently true generalization that is a sentence in the best system stated in terms of a given set of economic predicates. The advocates of BBSA emphasize that, for instance, the true deductive systems relative to the set $\mathbf{B}$ cannot be compared with the true deductive systems relative to the set $\mathbf{E}$. However, any true deductive system that is formulated in terms of the biological (and, respectively, the economic) predicates can be compared with any other system that is formulated in the same vocabulary. ${ }^{7}$

The strategy of relativizing a best system to a set of predicates has a pay-off: the BBSA permits to count special science generalizations as law statements despite the fact that their predicates refer to macroscopic, non-natural, non-fundamental properties (Schrenk 2007, 163; Cohen and Callender 2009, 24). Moreover, the BBSA aims to provide a unified theory of lawhood for all scientific disciplines. The laws of physics and the laws of biology are laws for the same reason - they are laws in virtue of being axioms (or theorems) of a best system relative to a chosen language. In this spirit, the general account of a law in the sciences reads as follow: a contingently true generalization is a law of a discipline $d$ iff it is an axiom (or a theorem) in the best system relative to the set of predicates $\mathbf{D}$ of discipline $d$. Cohen and Callender demand that the vocabulary used to formulate the systems should be stipulated according to the particular needs of scientific

7 Cohen and Callender propose a view called "explosive realism" about properties complementing the BBSA. According to explosive realism, "the world permits possibly infinitely ways of carving up into kinds, each equally good from the perspective of nature itself' (Cohen and Callender 2009, 22). That is, Cohen and Callender effectively deny the existence of Lewisian natural properties. One way of carving up the world differs from another by being "differentially congenial and significant to us given the kinds of creatures we are, perceptual apparatus we have, and (potentially, variable) matters we care about" (ibid.). 
practice. This view results in a multitude of best systems, each best in relation to the area of application (Cohen and Callender 2009, 22-24).

\section{Challenges to the Better Best Systems Analysis}

We will argue that the BBSA faces two challenges. Firstly, it is argued that BBSA faces Lange's Dilemma and it is at least questionable, or so we argue, whether BBSA can appeal to standard accounts of cp-conditions in order to avoid the dilemma. Secondly, we argue that BBSA as it stands cannot account for idealized laws.

\section{First Challenge: Lange's Dilemma and Ceteris Paribus Conditions}

Advocates of the BBSA take the law statements of the special sciences to be contingently true sentences. A law is a contingently true generalization that figures in a best system relative to some set of predicates. We do not object to this characterization of laws in the special sciences. However, in the debate on special science laws one cannot take it for granted that the law statements are contingently true. One reason to believe that one needs an argument for the claim that law statements are contingently true is Lange's dilemma (named after Marc Lange; Lange 1993, 235). ${ }^{8}$ Here is the first horn:

First horn (Falsity): Strictly and literally speaking, special science laws are false because it is not the case that all Fs are Gs (if that is what the laws say).

\footnotetext{
${ }^{8}$ We focus on Lange's dilemma in this section because it is one of the most influential problems in the recent literature on ceteris paribus laws. We turn to a distinct but related problem - Cartwright's dilemma - in the second challenge.
} 
For instance, consider the law of demand: under the condition of perfect competition, an increase of demand of a commodity leads to an increase of price, given that the quantity of the supply of the commodity remains constant (Reutlinger et al. 2011, section 1.1). The relationship between demand and price is not always as the law of demand says (or, as it seems to say prima facie), because an interfering factor might occur (as the supply may decrease, the government might interfere, a natural catastrophe might disturb the communication between buyers and sellers etc.). Special science laws that instantiate perfect regularities are "scarce" (Cartwright 1983, 45) because disturbing factors do in fact occur - and, in some cases, quite frequently so. If a law statement is formalized as a universally quantified conditional, then one counter-instance (due to a single disturbing factor) to the universally quantified sentence suffices to evaluate the sentence as false.

The second horn of Lange's Dilemma can be stated as follows:

Second horn (Triviality): If special science law statements are qualified by ceteris paribus conditions, then these law statements are trivially true.

Suppose that a ceteris paribus clause is attached to a law to the effect that the ceteris paribus law statement is equivalent to "All Fs are Gs, if nothing interferes". Then the ceteris paribus law in question seems to be void of empirical content, because it seems to say "All Fs are Gs or (it is not the case that all Fs are Gs)". Note that the second horn of Lange's dilemma seems to depend on the so-called exclusive reading of the ceteris paribus clause ("if nothing interferes", "if all disturbing factors are absent"). If this were 
the correct theory of laws in the special sciences, then these laws would be analytically true sentences and, therefore, trivially true. This is a bad result because a vast majority of philosophers is convinced that laws of the special sciences ought to be reconstructed as non-trivially true statements.

We focus on Lange's dilemma in the present section because it is one of the most influential problems in the recent literature on ceteris paribus laws. We turn to a distinct major problem - Cartwright's dilemma - in the second challenge. Lange's and Cartwright's dilemma differ in the following way: Lange's dilemma states that some law statements are either false statements or analytically true statements. According to Cartwright's dilemma, some law statements are either false statements, or vacuous empirical statements (that is, the latter are not analytic truths). Hence, the dilemmas differ in how they contrast falsity in the second horn: falsity is contrasted with analytic truth in Lange's dilemma; falsity is contrasted with empirical vacuity in Cartwright's dilemma.

Since Lange's dilemma is a well-known challenge to any theory of laws in the special sciences, it is reasonable to expect that the BBSA - qua theory of special science laws - provides a strategy to avoid Lange's dilemma. It is a surprising fact that Cohen and Callender do not live up to this expectation. They simply take it as a fact that the laws of the special sciences are true and non-trivial. Cohen and Callender seem to get the dialectic wrong when they briefly address the second horn of the dilemma: "the thought is that exceptionable generalizations are non-vacuous only if they are part of the best system [...]" (Cohen and Callender 2009, 25). It is correct to say that, according to the BBSA, every law candidate has to be a true empirical statement in order to play a role in a best system. However, since Lange's dilemma jeopardizes precisely this claim, 
advocates of BBSA ought to explain why laws in the special science are empirical and true statements. Lange's dilemma is a rather specific problem for theories of special science laws, since these laws tend to have exceptions. An account of laws of physics might ignore Lange's dilemma assuming that fundamental laws do not have exceptions, but defenders of BBSA qua theory of special science laws have to show how the dilemma can be avoided. To be fair to Cohen and Callender, it is worth noting that they are aware of the challenge that Lange's dilemma poses. In a brief footnote, Cohen and Callender endorse the claim that the BBSA is compatible with various accounts of ceteris paribus laws: "we won't pick an option here; suffice to say that there are some" (Cohen and Callender 2009, note 24). However, they do not show that this is in fact true. It is questionable whether their claim is true, as we will argue. However, to forestall misunderstandings, our concern is not merely that proponents of the BBSA do not in fact propose a strategy to avoid Lange's dilemma. We are concerned with the question whether the BBSA is compatible with attaching "non-lazy" cp-clauses to the axioms and theorems of the best system. ${ }^{9}$

To sum up our initial concern, Lange's dilemma is a challenge that all accounts of (special science) laws face. We would like to stress that it does not suffice to gesture towards potential strategies of dealing with Lange's dilemma. Instead proponents of the BBSA have to show that these strategies are available to them. Dealing with this challenge is especially pressing in the context of special science laws, because special

${ }^{9}$ Ceteris paribus clauses may also qualify statements that are not law statements. Reutlinger et al. (2011, section 9) provide a survey to the usage of ceteris paribus conditions in other areas of philosophy than philosophy of science (e.g. ethics, formal semantics, formal epistemology, and philosophy of language). Nickel (forthcoming) discusses the relation between cp-conditions and generic conditionals in philosophy of language. Our present discussion is restricted to ceteris paribus clauses as applied to law statements. 
science laws are subject to cp-clauses.

Unlike Cohen and Callender, Schrenk (2007, 162-171) proposes an elaborate strategy to integrate cp-clauses into the BBSA (also Schrenk forthcoming, section 3). Schrenk holds that it is possible to interpret a special science law as contingently true if all actual exceptional individuals to the generalization are explicitly listed. More precisely, Schrenk proposes to read 'ceteris paribus, $\forall \mathrm{x}$ : if $\mathrm{Fx}$, then $\mathrm{Gx}$ ' as ' $\forall \mathrm{x}$ : if $\mathrm{Fx}$ except for the individuals $a, b$, $c$, etc., then Gx'. Schrenk uses space-time points and tigers as examples of individuals. To take Schrenk's own example, 'ceteris paribus, $\forall \mathrm{x}$ : if $\mathrm{x}$ is a tiger, then $\mathrm{x}$ has orange and black stripes' ought to be understood as ' $\forall \mathrm{x}$ : if $\mathrm{x}$ is a tiger, except for Bino the albino tiger, then $\mathrm{x}$ has orange and black stripes'. Schrenk uses a metaphor in order illustrate his account of ceteris paribus laws. Imagine that the best system for a given vocabulary is determined from a divine perspective. An omniscient divine being which possesses complete knowledge of the universe could provide us with a best system for any set of predicates that refer, if nature is kind to us - to use Lewis's expression - and nature can be systematized by a best system at all. This divine being would also be able to complete the universal laws with a full list of all individual exceptions. If such a proposition still fits into the best system, then it is a law. Schrenk argues that "ceteris paribus" is an abbreviation for the list of all individual exceptions to a law:

This cosmetic operation is less superficial than it might seem: [...] it translates the lengthy statements that have participated in the [best system] contest into the law statements that are typical for the special science: law 
statements with proviso [here synonymously used with 'ceteris paribus']

clauses. (Schrenk 2007, 166)

According to Schrenk, a law statement (including a list of exceptions) may figure in a best system, it may be contingently true and, hence, avoid Lange's dilemma. Call this view Schrenk's 'exception-account'. Although we applaud Schrenk's general attempt of addressing Lange's Dilemma and of providing an account of ceteris paribus conditions complementing the BBSA, we are skeptical about Schrenk's proposal. We raise two objections to Schrenk's exception-account.

Although Schrenk's account succeeds in avoiding Lange's dilemma, the exception-account does not satisfactorily account for ceteris paribus laws. Consider Schrenk's example of a ceteris paribus law statement ' $\forall \mathrm{x}$ : if $\mathrm{x}$ is a tiger, except for Bino the albino tiger (etc.), then $\mathrm{x}$ has orange and black stripes'. We grant Schrenk that this sentence is neither trivially true nor contingently false. However, the exception account does not provide an answer to the pressing question why or in virtue of what there are these exceptions. Even if it is true that Bino the albino tiger is an exception to the law that all Tigers are stripy, we expect from an account of ceteris paribus conditions that exceptional individuals are not merely listed, but that the ceteris paribus clause provides some non-trivial qualitative information about the condition(s), under which an exception occurs. That is, following Pietroski and Rey's $(1995,89)$ intuition, we would like to know more than just the fact that Bino is exceptional; what we really want to know it why there are exceptions like Bino and, particularly, what it is about Bino - the internal and external conditions he is in - that results in an exception to the law in question. We 
believe that it is fair to say that such a 'qualitative' characterization of conditions leading to a counter-instance to a law (that is, ceteris paribus conditions) is a generally accepted condition of adequacy for an account of ceteris paribus laws (cf. Earman and Roberts, 1999; Reutlinger et al., 2011). Schrenk's exception-account apparently does not meet this condition of adequacy.

Suppose one accepts the demand for a qualitative characterization of exceptional conditions; the majority of accounts of cp-laws respect this demand. Then, we anticipate the following question: even if Schrenk's account of cp-laws fails, are there other accounts of cp-laws that might complement the BBSA? One possible alternative to Schrenk's exception-account is Jerry Fodor's (1991) completer account. ${ }^{10}$ Fodor's account responds to the need of getting a qualitative characterization of cp-conditions. The core idea of Fodor's completer account is that 'ceteris paribus, $\forall x$ : if Fx, then Gx' should be read as ' $\forall \mathrm{x}$ : if $\mathrm{Fx}$ and $\mathrm{Cx}$, then Gx' - where the completer $\mathrm{C}$ is a set of nonredundant conditions that is, in conjunction with the antecedent, sufficient for the consequent. By adopting this strategy instead of Schrenk's, it becomes possible to provide a qualitative characterization of exceptional conditions (that is, whether or not the law holds depends on the presence of conditions C). However, one problem - for the BBSA - with Fodor's approach is that the completer for, say, Schrenk's tiger-law, or a law of economics, often is some physical condition. It is important to remember that the advocates of the BBSA propose a multitude of best systems, one for each special science, each with its own more or less distinct vocabulary. If we suppose that some completers of the tiger-law, or an economic law, are physical (or chemical or biological), then there is

${ }^{10}$ Reutlinger et al. (2011) provide a survey to recent improved versions of completer accounts. 
no guarantee that the language of a given special science contains the physical predicates required to pick out the completer. Thus, if the best system containing the tiger-law is relative to a set of biological properties only, then an advocate of BBSA cannot straightforwardly adopt Fodor's completer account. ${ }^{11}$

One might wonder (as a referee did) whether the need to "go beyond" the vocabulary of some special science $\mathrm{S}$ in order to spell out the cp-clause for a law of $\mathrm{S}$ is an idiosyncratic feature of Fodor's completer account. We believe that this is not the case. The need to 'go beyond' the vocabulary of one special science, such as biology, in order to characterize (a possibly open-ended list) of ceteris paribus conditions is not a specific feature of Fodor's approach. It is a general characteristic of many accounts of ceteris paribus laws, including completer accounts, normality accounts, dispositionalist accounts (Earman and Roberts, 1999; Reutlinger et al., 2011; Reutlinger forthcoming). We side with Earman and Roberts (1999) who take this "going beyond" feature to be essential for genuine ceteris paribus laws. Earman and Roberts $(1999,462-463)$ refer to this feature as the "non-lazy" character of ceteris paribus conditions: cp-conditions of a law of special science S are non-lazy iff the cp-conditions are (partly) outside of the conceptual and methodological scope of S and the list of conditions is potentially openended. If cp-conditions are non-lazy, then the feature of non-laziness alone independently of a specific account of cp-conditions - raises a general problem for the BBSA that is exemplified by the completer account: accounting for cp-conditions of special science laws at least in some cases requires to expand a best system's vocabulary beyond the special science in question. That is, a cp-law of economics is ultimately not

${ }^{11}$ Schrenk $(2007,168)$ briefly refers to Pietroski and Rey's (1995) completer account. However, a proponent of the BBSA faces the same difficulty in the case of Pietroski and Rey's approach as with Fodor's account. 
an axiom of a best system formulated in purely economic vocabulary. If a law of economics is a cp-law, then it is an axiom of a best system formulated in terms of economic predicates, while its cp-conditions are expressed using biological and physical predicates. $^{12}$

Why is expanding the vocabulary a problem? One reaction might be to simply accept that one need to extend the set of properties (relative to which a best system is formulated) in order to capture the ceteris paribus conditions adequately: for instance, the best system for biological laws is relative to biological as well as chemical and physical properties (see Frisch forthcoming). However, this strategy faces the problem of supervenient properties (see section 2). According to Cohen and Callender, this problem is put as follows: for a deductive system that contains both higher-level and lower-level generalizations, it is the case that the higher-level laws are redundant; or, if the higherlevel laws are translated into the lower-level language, the higher-level laws unnecessarily complicate the system (because higher-level properties are multiply realized) and, therefore, cannot count as laws. Recall that to avoid the problem of supervenient properties is one of the motivations of proposing a BBSA. If Cohen and Callender wish to attach "non-lazy" ceteris paribus clauses to generalizations in a better best system, then it is far from clear how they could avoid the problem. One way out for proponents of the BBSA might be to drop the problem of supervenient properties (for

12 Moreover, it does not suffice to simply add "non-lazy" predicates to a best system in order to properly account for cp-conditions of a given law of that system. In our example, in order to know why biological and physical predicates are relevant for the exceptions to a cp-law in economics, we need these predicates to figure in laws of the best systems for biology or physics, which account for the conditions that are relevant for the exceptions to the economical cp-law. Additionally, one would also need to enrich the vocabulary of a given special science by bridge principles spelling out these intertheoretic connections between different systems. 
instance, by denying that higher-level laws are redundant). But then it remains to be shown how the whole project of the BBSA gets off the ground at all.

One might worry (as a referee suggested) that the problem of supervenient properties does not arise if one rejects natural properties. However, this is not necessarily the case, because there might still be 'systematic inter-theoretic relationships' between the best systems of different sciences (for instance, relationships such as theory reduction, supervenience of one discipline's Humean mosaic on the mosaic of another discipline another, grounding, etc.). Cohen and Callender, for instance, emphatically endorse the claim that - "if we have reason to believe in anything in science" - macroscopic physical properties (and other special science properties) supervene on microscopic features of physical systems (Callender and Cohen 2010, 432). Moreover, Cohen and Callender explicitly reject Cartwright's view of a 'dappled world' whose core is the denial of such 'systematic inter-theoretic relationships', particularly, the denial of supervenience relations (Callender and Cohen 2010, 431). If there are systematic relationships holding between (the posits of) better best systems (such as supervenience relations), then the problem of supervenient properties may arise even if one rejects natural properties. Interestingly, Frisch (forthcoming) provides a critical discussion of the alleged redundancy of special science generalizations in the framework of the BBSA. However, our focus is on Cohen and Callender's view that the problem of supervenient properties is indeed a problem.

To conclude, the main point we stress in this section is that avoiding Lange's dilemma cannot be accomplished by the means of the BBSA alone. Callender, Cohen and Schrenk need an additional tool in their theoretical apparatus to do this: an account of 
ceteris paribus clauses or, more generally speaking, an account of the non-strict, exception-ridden character of special science laws. Without such a complementary account, BBSA seems to be an incomplete theory of special science laws. Schrenk's exception-account is flawed. We presented an argument to the conclusion that other accounts to ceteris paribus conditions are not compatible with the BBSA - in particular, non-lazy cp-conditions are not compatible with avoiding the problem of supervenient properties.

\section{Second Challenge: Cartwright's Dilemma and Idealized Laws}

Our second objection to the BBSA is motivated by Cartwright's dilemma. This dilemma is based on the observation that special science laws often include idealizations, i.e. intentionally distorted, non-veridical representations of reality. ${ }^{13}$ According to Cartwright's dilemma, many special science laws are either literally false or vacuously true because they are idealized. For instance, economic laws often assume perfect competition - that is, the assumption that every agent on a market has complete information, transitive preferences, and the like. Take the law of demand as example of an idealized law: under the condition of perfect competition, an increase of demand of a commodity leads to an increase of price, given that the quantity of the supply of the commodity remains constant. Strictly speaking, the condition of perfect competition is

\footnotetext{
${ }^{13}$ Cartwright's dilemma does not only affect the BBSA but every realist conception of laws that implies that law statements must be true - for instance, theories of laws of nature by Armstrong 1983, Lewis 1994, Maudlin 2007, and Lange 2009. The fact that other accounts suffer from the same problem does not diminish our criticism that an account of special science laws should be able to deal with the problem that Cartwright's dilemma poses. Moreover, we will argue below that received strategies for avoiding Cartwright's dilemma are not available for a proponent of the BBSA.
} 
never, or extremely rarely, satisfied in real markets. For this reason it may be argued that the law of demand is either false for real agents; or the law is vacuously true because it has no - or, at best, very few - instances and only applies to ideal agents (Cartwright 1983, 47; Pietroski and Rey 1995, 84). Hence, the idealized law seems to be either false or vacuously true. Since this dilemma is most forcefully presented in Cartwright (1983), we call it 'Cartwright's dilemma'. Cartwright's dilemma is in conflict with the assumption that law statements are true and have a considerable degree of strength, as proponents of best system approaches generally believe. ${ }^{14} \mathrm{We}$ argue that an adequate theory of laws in the special sciences ought to count idealized laws as laws, but the BBSA fails to satisfy this condition of adequacy.

Our argument for why BBSA fails to account for idealized special science laws is straightforward. By definition, a deductive system includes only those contingently true generalizations that offer the best balance of empirical strength and simplicity. According to Cartwright's dilemma, idealized laws are either false or they lack strength (because they are vacuously true). We argue that advocates of the BBSA have to choose between two options: (i) either idealized laws are not laws according to the BBSA. In this case, the BBSA fails to be an adequate account of laws in the (special) sciences, as it does not apply to statements that the (special) sciences, in fact, treat as laws. (ii) Or a proponent of the BBSA drops the requirement that law statements need to be true statements - that is,

${ }^{14}$ As a referee correctly pointed out, there could be best systems that are extremely simple but have very little strength, yet are still overall optimally balanced. However, the law statements in the sciences describing the actual world typically are well suited for prediction and explanation and, hence, possess a high score on strength. This is no accident: it is a common view that scientists accept theories because of their "considerable" predictive and explanatory strength. However, all we need to claim for the purpose of this argument is that actual law statements seem to have some degree of empirical strength, i.e. they are not empty. 
the axioms and theorems of the best system may be literally false statements if they offer the best balance of simplicity and strength.

On the one hand, if one considers idealized laws to be false, then this strategy is in contradiction to the demand that that all of the axioms and the theorems of the best system need to be true. In response, a proponent of the BBSA might abandon the requirement that the axioms and theorems of the best system have to be literally true and allow false statements as laws if they contribute to the best balance of simplicity and strength. David Braddon-Mitchell (2001) defends such a non-standard 'instrumentalist' version of the BSA, which is compatible with accepting the falsity of idealized laws (see also Frisch [forthcoming]). However, we are discussing the BBSA (as advocated by Callender, Cohen, and Schrenk), which agrees with the original BSA on the demand that law statements need to be true. On the other hand, adding a vacuously true statement to a deductive system results in a decrease of simplicity without any (or very little) gain in empirical strength. Therefore, idealized laws qua vacuous laws do not figure in the best system.

Admittedly there is one exception: the axioms of a best system might entail a vacuously true generalization as a theorem, which then counts as a law (Loewer 1996: 111). Newton's first law of motion might be an example. Although many philosophers take Newton's first law to be an axiom (cf. Maudlin 2007, chapter 5; Maudlin 2012, chapters 1 and 2; Hüttemann 2004, chapter 2; Hüttemann, forthcoming; Smith, 2002; Smith, 2008), Newton's first law, arguably, might be regarded as a theorem, as a limiting case of the second law. ${ }^{15}$ We do not deny that this story applies to some vacuous

15 It seems, however, to be the consensus in the literature that Newton's first law is regarded not to be a consequence of the second law, at least not in its historic form (cf. 
generalizations. But we doubt that it amounts to a general theory of idealized laws in the special sciences, because many idealized special science laws seem to be axioms rather than theorems of special science theories or models.

Advocates of the BBSA might reply that one could avoid our objection by separating the task of providing an account of laws and the task of making sense of idealizations. One option to make sense of idealized laws is to say that an idealized law statement is not literally true but such a law describes how non-ideal entities approximately behave. There are at least two notorious problems with understanding idealization in terms of approximation. The first problem is to make precise the concept of 'approximation' or 'approximate truth'. The critics hold that the notion of approximation is prima facie not helpful to improve our understanding of idealizations, because the notions of approximation and approximate truth stand in as much need of clarification as the notion of idealization does (cf. Stanford, 2006; Strevens, 2008).

Secondly, even if one grants that we have a satisfactory grasp of the notion of approximation, some philosophers convincingly argue that the approximation account of idealization does not cover all cases of idealized laws (cf. Weisberg, 2007; Strevens, 2008). Proponents of the BBSA might respond that they could adopt alternative accounts of idealization, if the approximation account of idealization fails. For instance, advocates of the BBSA might wish to adopt the dispositionalist approach to idealized laws (cf. Cartwright, 1983; Hüttemann, 1998). A dispositionalist holds that idealized laws are the statements about the behavior that an entity would manifest if this entity were

Maudlin 2007, 155; Cohen 2002, 68-70; Earman and Friedman 1973, 345-346). We are grateful to an anonymous referee for drawing our attention to this point. If it is indeed correct that the first law is an axiom, then all the worse for the proponents of the BBSA: then, the stance that (a) vacuous statements are mere theorems of the best system and that (b) Newton's first law is a paradigm case of such a theorem loses its initial plausibility. 
undisturbed by or isolated from disturbing factors. But the BBSA as a Humean project is incompatible with this interpretation of idealizations, because the dispositionalist approach - at least in its standard formulation - accepts irreducible dispositions (Cartwright, 1989; Hüttemann, 1998; Bird, 2007). Even if we grant that there is an antiHumean version of $\mathrm{BBSA}^{16}$, the dispositionalist account refers us back to original problem that we introduced earlier: for the dispositionalist an idealized laws is a claim about the behavior of an undisturbed or isolated system. However in the framework of the BBSA, such a claim seems to have no instances and, as it does not summarize actual regularities, lacks empirical strength. Note that this is per se not a problem for the dispositionalist but it is a problem for a proponent of the BBSA. As argued above, the lack of strength precludes an idealized claim from figuring in the best system. Hence the dispositionalist account does not help proponents of the BBSA. ${ }^{17}$

Similar remarks apply to Strevens's kairetic difference-making account of idealized laws (Strevens 2008, chapter 8). The gist of Strevens's view is that idealizations provide information about which factors are explanatorily irrelevant (that is, which factors are not "difference-makers"). If proponents of the BBSA were to adopt Strevens's account of

${ }^{16}$ For instance, Demarest (forthcoming) elaborates an anti-Humean BSA. This view is compatible with the BBSA, since Cohen and Callender (2009, 3, note 1) point out that, although they are partly attracted to the BBSA because it is compatible with a broadly Humean metaphysics, Humean supervenience and best system accounts are "logically distinct"; see also Callender and Cohen (2010, 432 and 433, note 6).

${ }^{17}$ Another option (that an anonymous referee suggested) for a dispositionalist account of idealized special science laws could be to claim that idealized laws describe the net behavior of a given system, which could possibly even be analyzed in terms of reducible dispositions. If these dispositions are taken to be reducible, the proposal remains firmly on Humean grounds. However, we are skeptical as to whether such a reductive account ("the conditional analysis") can be defended. Moreover, it remains to be shown whether the conditional analysis of dispositions can cope with the fact that, in the context of idealized laws, the stimulus conditions only hold for ideal systems or the conditional in question seems to be false. In the literature, cases involving idealizations and cpconditions are exactly those cases in which the conditional analysis fails (Bird, 2007). 
explanatory relevance in order to interpret the content of idealized law statements, then this strategy would raise two problems. Both problems concern the question what is taken to be fundamental in metaphysical and conceptual accounts of laws. Callender and Cohen seem to believe that nomic facts are more fundamental than causal facts and facts about what explanations are. This belief is in tension with applying Strevens' account of idealizations to the BBSA. Firstly, since Strevens advocates a causal model of explanation and if this causal model is used to interpret the content of idealized law statements, then causal facts would seem to be more fundamental than nomic facts. Taking causal facts as more fundamental than nomic facts is first and foremost at odds with a Humean account, and it is also at odds with several anti-Humean accounts of laws (for instance, Maudlin, 2007), and the claim that causation is not fundamental in the light of presently accepted physical theories (Price and Corry, 2007). Secondly, given their (Humean) belief that nomic facts are more fundamental than causal facts and facts about what explanations are, Callender, Cohen and Schrenk should not base their theory of idealized (special science) laws on a theory of causal explanation. If they did, this would amount to taking causal explanations to be (conceptually or ontologically) more basic than the laws instead of using the laws to explicate what a scientific explanation is. Loewer (1996), to whom Cohen and Callender approvingly refer, considers it to be one of the prime virtues of best system accounts of laws that it elucidates how laws can figure in scientific explanations.

\section{Conclusion}

Callender, Cohen, and Schrenk's BBSA is attractive because it promises to be a unified 
theory of laws - that is, a theory according to which lawhood in physics and lawhood in the special sciences is explicated by the same philosophical account. We have presented two challenges to the BBSA, both of which are concerned with the question whether BBSA is an adequate and convincing theory of special science laws. We argue that BBSA cannot avoid Lange's dilemma (first challenge). Moreover, BBSA fails to account for idealized laws (second challenge). In order to be an adequate theory of special laws, BBSA has to deal with these desiderata.

\section{ACKNOWLEDGEMENTS}

We would to thank Craig Callender, Jonathan Cohen, Laura Franklin-Hall, Mathias Frisch, Andreas Hüttemann, Siegfried Jaag, Stathis Psillos, John T. Roberts, Markus Schrenk, the audiences of two talks at the University of Cologne, and three anonymous referees for productive comments on earlier drafts of the paper. Marius Backmann is very grateful to the Netherlands Organization for Scientific Research (NWO) for their funding within the framework of the project "What is Really Possible?", NWO VIDI 276-20-013.

\section{REFERENCES}

AlBert, D. 2000, Time and Chance, Cambridge, MA: Harvard University Press.

Armstrong, D. 1983, What is a Law of Nature?, Cambridge: Cambridge University Press.

BIRD, A. 2007, Nature's Metaphysics, Oxford: Oxford University Press.

BRaddon-Mitchell, D. 2001, “Lossy Laws”, Noûs 35, pp. 260-277. 
Callender, C. and Cohen J. 2010, "Special Sciences, Conspiracy and the Better Best System Account of Lawhood", Erkenntnis 73, pp. 427-447.

Cartwright, N. 1983, How the Laws of Physics Lie, Oxford: Oxford University Press.

Cartwright, N. 1989, Nature's Capacities and Their Measurement, Oxford: Oxford University Press.

CoHEN, B. 2002, 'Newton's Concepts of Force and Mass, with Notes on the Laws of Motion", In: I.B. Cohen and G.E. Smith, eds., The Cambridge Companion to Newton, Cambridge: Cambridge University Press, pp. 57-84.

Cohen, J. and Callender, C. 2009, "A Better Best System Account of Lawhood”, Philosophical Studies 145, pp. 1-34.

Demarest, H. forthcoming, "Powerful Properties, Powerless Laws”, in: J. Jacobs, ed., Putting Powers to Work: Causal Powers in Contemporary Metaphysics, Oxford: Oxford University Press.

EARman, J. and Friedman, M. 1973, “The Meaning and Status of Newton's Law of Inertia and the Nature of Gravitational Forces", Philosophy of Science 40, pp. 329-359.

Earman, J. and Roberts, J.T. 1999, "Ceteris Paribus, there is no Problem of Provisos", Synthese 118, pp. 439-478.

Fodor, J. 1991, "You Can Fool Some People All of the Time, Everything Else Being Equal; Hedged Laws and Psychological Explanations”, Mind 100, pp. 19-34.

Frisch, M. forthcoming, "Why Physics Can’t Explain Everything”, in: A. Wilson, ed., Asymmetries of Time and Chance, Oxford: Oxford University Press.

Hüttemann, A. 1998, “Laws and Dispositions”, Philosophy of Science 65, pp. 121-135. 
Lange, M. 1993, "Natural Laws and the Problem of Provisos", Erkenntnis 38, pp. 233248.

LANGe, M. 2009, Laws and Lawmakers. Science, Metaphysics, and the Laws of Nature, Oxford: Oxford University Press.

LEwIS, D. 1973, Counterfactuals, Oxford: Oxford University Press.

LEwIS, D. 1983, "New work for a theory of universals", in: D. Lewis, Papers in Metaphysics and Epistemology, Cambridge: Cambridge University Press, pp. 855.

LEwIS, D. 1994, "Humean Supervenience Debugged", in: D. Lewis, Papers in Metaphysics and Epistemology, Cambridge: Cambridge University Press, pp. 224-247.

Loewer, B. 1996, "Humean Supervenience”, Philosophical Topics 24, pp. 101-127.

Loewer, B. 2007, “Laws and Natural Properties”, Philosophical Topics 35, pp. 313-327.

Loewer, B. 2009, “Why Is There Anything Except Physics?”, Synthese 170, pp. 217233.

Maudlin. T. 2007, The Metaphysics Within Physics, New York: Oxford University Press.

Maudlin, T. 2012, Philosophy of Physics. Space and Time, Princeton: Princeton University Press.

Nickel, B. forthcoming, "The Role of Kinds in the Semantics of Ceteris Paribus Laws", Erkenntnis.

Pietroski, P. and Rey, G. 1995, “When other Things aren't Equal: Saving Ceteris Paribus Laws from Vacuity", British Journal for the Philosophy of Science 46, pp. 
$81-110$.

Price, H. and Corry, R. (eds.) 2007, Causation, Physics, and the Constitution of Reality. Russell's Republic Revisited, New York: Oxford University Press.

Reutlinger, A. forthcoming, "Do Statistical Laws Solve the Problem of Provisos?", Erkenntnis.

Reutlinger, A., Schurz, G. and Hüttemann, A. 2011, "Ceteris Paribus Laws”, The Stanford Encyclopedia of Philosophy (Spring 2011 Edition), Edward N. Zalta (ed.), URL = <http://plato.stanford.edu/archives/spr2011/entries/ceterisparibus/>.

Schrenk, M. 2007, The Metaphysics of Ceteris Paribus Laws. Frankfurt: ontos.

SchrenK, M. 2008, “A Theory for Special Science Laws”, in: H. Bohse, K. Dreimann and S. Walter, eds., Selected Papers Contributed to the Sections of GAP.6, 6th International Congress of the Society for Analytical Philosophy. Paderborn: Mentis.

SCHRENK, M. forthcoming, "Better Best Systems and the Issue of CP-Laws", Erkenntnis.

SCHRENK, M. manuscript, "Properties for and of Better Best Systems".

Smith, G. 2008, “Newton's Philosophiae Naturalis Principia Mathematica, The Stanford Encyclopedia of Philosophy", (Winter 2008 Edition), Edward N. Zalta (ed.), URL $=<$ http://plato.stanford.edu/archives/win2008/entries/newton-principia/ $>$.

SMITH, S. 2002, "Violated Laws, Ceteris Paribus Clauses, and Capacities”, Synthese 130: pp. 235-264.

StANFoRD, K. 2006, Exceeding Our Grasp, New York: Oxford University Press.

Strevens, M. 2008, Depth. An Account of Scientific Explanation, Cambridge, MA: 
Harvard University Press.

Strevens, M. forthcoming, "Ceteris Paribus Hedges: Causal Voodoo That Works", Journal of Philosophy.

VAn FraAssen, B. 1989, Laws and Symmetries, Oxford: Clarendon Press.

WeIsBerg, M. 2007, “Three Kinds of Idealization”, The Journal of Philosophy 104: pp. 639-59. 